\title{
Photoluminescence of ZnSe nanocrystals at high excitation level
}

\author{
V.V. Tishchenko \\ Institute of Physics of the National Academy of Sciences of Ukraine, 46 Nauki Ave., Kyiv 03028, Ukraine \\ E-mail: vvti@iop.kiev.ua
}

\author{
A.V. Kovalenko \\ Dnipropetrovsk National University, 13 Naukova Str., Dnipropetrovsk 49050, Ukraine \\ E-mail:koval@mail.dsu.dp.ua
}

Received December 25, 2008, revised January 12, 2009

\begin{abstract}
The excitation-dependent photoluminescence (PL) of ZnSe nanocrystals (NC) grown on GaAs (100) substrate was studied. The PL spectra observed corroborate previous observations of a bimodal size distribution of $\mathrm{NC}$ grown, and, in addition, evidence the existence of spectral diffusion with extend dependent on excitation power. Besides, it was also shown that at relatively intense excitation an extra band has arose in luminescence spectra due to biexcitons confined in NC of 3.5-4.0 nm size. The binding energy of these biexcitons was as large as $23 \mathrm{meV}$.
\end{abstract}

PACS: 78.67.Bf Nanocrystals and nanoparticles;

78.67.Hc Quantum dots;

78.55.Et II-VI semiconductors.

Keywords: quantum dots, nanocrystals, biexciton, photoluminescence, $\mathrm{ZnSe}$.

In recent years the properties of highly excited semiconductor nanocrystals (NC) widely referred to as semiconductor quantum dots (QD) have gained much attention largely due to advance in application development motivated by the demonstration of effective lasing in CdSe-based QD [1]. At the same time the semiconductor compound which has received the most intense interest in previous years has been $\mathrm{ZnSe}$ known to have a room temperature direct band gap of $2,7 \mathrm{eV}$ that corresponds to emission in the blue region of the spectrum. Properly tailored ZnSe-based QD are well suited for short wavelength light emitters, a fact recognized worldwide by research groups several of whom have expanded a great deal of effort attempting to produce $\mathrm{ZnSe} \mathrm{NC}$ of high quality $[2,3]$. However properties of extremely small $\mathrm{ZnSe}$ crystals under intense optical excitation remain unexplored and require better insight.

Recently, a vapor phase technique has been utilized to grow ZnSe nanocrystals atop GaAs (100) substrate [4]. The distinguishing feature of applied technique was the use of horizontal type quartz reactor exploiting a large $\left(\sim 250^{\circ} \mathrm{C} / \mathrm{cm}\right)$ temperature gradient in the deposition zone just in front of substrate on which ZnSe NC were collected. Applied characterization techniques indicated the formation of two distinct sets of nanocrystals on the same substrate. The average characteristic sizes of NC in these sets have been estimated to be in the ranges of 36.6-41.2 and 3.5-4.0 nm while the effective Bohr radius $\left(a_{B}\right)$ of the free exciton in bulk ZnSe is known to be $\sim 50 \AA$ [5]. From these facts one can easily realize that samples described in Ref. 4 are unique since they provide chance to compare behavior of carriers confined in sub-excitonic and superexcitonic volumes of $\mathrm{ZnSe}$ by studying photoluminescence (PL) just from the same single sample. The present work extends research on those samples with the aim to investigate their emission spectra under high level of optical excitation.

The excitation source was $\mathrm{N}_{2}$ laser $(337.1 \mathrm{~nm})$ with optical pulses of $10^{-8} \mathrm{~s}$ and $100 \mathrm{~Hz}$ repetition rate. In our case this excitation can be treated as a quasi-continuous-wave (qCW) excitation as the pulses used were long enough compared to all characteristic times known for nonequilibrium e-h system in direct-gap semiconductors. 
The parallel-sided quartz plate was used to split laser beam into excitation and reference parts. To measure the luminescence at different excitation intensity $\left(I_{\text {exc }}\right)$ the excitation split-off was guided to sample via calibrated neutral filters (attenuator) and focused on the surface of sample either loosely or exactly (the spot diameter was either $10^{3} \mu \mathrm{m}$ (loose focusing) or $\sim 10^{2} \mu \mathrm{m}$ (exact focusing)). Attenuator has allowed to readjust the value of $I_{\text {exc }}$ over about two orders of magnitude. At the same time the initial amplitude of the laser pulses was continuously monitored with photodiode exposed to reference split-off.

During the measurements the samples were mounted inside an optical cryostat operating in nitrogen-immersion mode. A computer controlled digital registration system featured step-by-step spectral scanning and gated detection of PL intensity relevant to maximum of excitation pulse. In the course of on-line processing only data corresponded to excitation pulses with less than $\pm 5 \%$ amplitude deviation where accumulated.

Figure 1 presents typical picture of the emission of the studied samples under two excitations of different kind: loosely focused $\mathrm{qCW}$ excitation and true $\mathrm{CW}$ excitation exploited in Ref. 4. Even the passing glance evidences the similarity between two spectra in Fig. 1. They include $S$ band originated from transitions relevant to the $S$ set of nanocrystallites that is to the set with the smaller representative size and $L$ band which is thought to be originating from the oversized $L$ set. We found that at $\mathrm{CW}$ excitation ratio of peak intensities of these bands varies slightly from sample to sample. Also a faint sample-to-sample variation was observed for the emission center $\left(\lambda_{0}^{S}\right)$ of the $S$ band whereas such peculiarity for the $L$ band was not detected. Taking into account the weak dependence of $\lambda_{0}^{S}$ on excitation sport position it is plausible to attribute observed sample-to-sample dependences to the inhomogeneity of the samples under the study. Due to contribution of quantum confinement such inhomogeneity will affect in the first place the spectral position of the $S$ band.

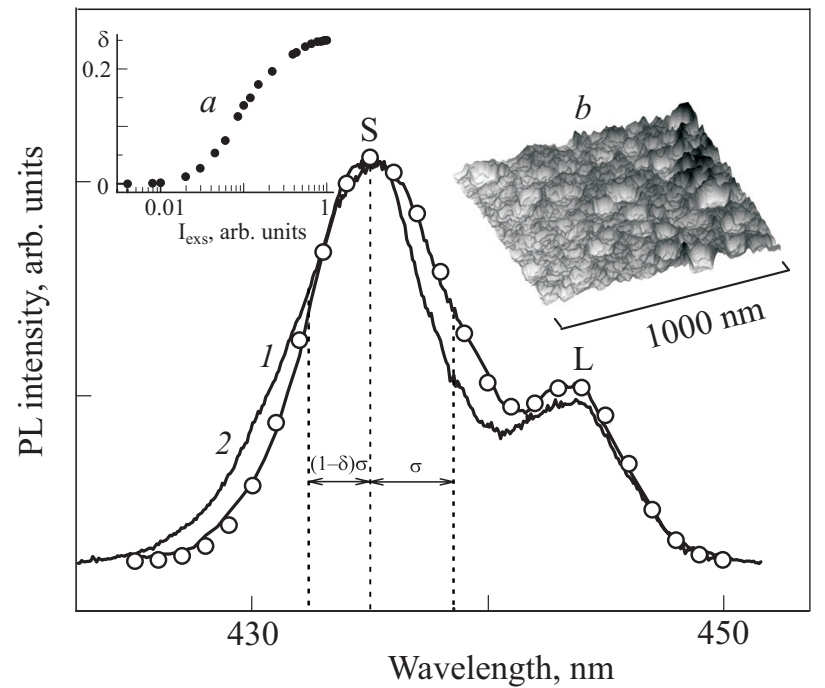

Fig. 1. PL spectra of $\mathrm{ZnSe}$ nanocrystals under CW (1) and loosly focused $\mathrm{qCW}(2)$ excitation at $77 \mathrm{~K}$. Both spectra has been normalized to the intensity of $S$ band. In the case of $\mathrm{CW}$ excitation $\left(325 \mathrm{~nm}\right.$ line of $10 \mathrm{~mW} \mathrm{He}-\mathrm{Cd}$ laser) $I_{\text {exc }}$ corresponds to $\sim 4 \cdot 10^{17}$ quanta $/\left(\mathrm{cm}^{2} \cdot \mathrm{s}\right)$, while in the case of $\mathrm{qCW}$ $I_{\text {exc }} \sim 10^{20}$ quanta/ $\left(\mathrm{cm}^{2} \cdot \mathrm{s}\right)$. Open circles are result of fitting of spectrum 2 with $\alpha_{j} \Gamma^{j}$ function, $j=S, L ; \alpha_{j}$ is the numerical parameter; $\delta \equiv \delta^{S}$ and $\sigma \equiv \sigma^{S}$. Inset $a$ shows a semilogarithmic plot for dependence of asymmetry parameter of $S$ band on $I_{\text {exc }}$ normalized to value of $10^{20}$ quanta/ $\left(\mathrm{cm}^{2} \cdot \mathrm{s}\right)$, while inset $b$ illustrates surface of the sample with given PL spectra.

Noteworthy is the absence of any new bands for any excitation spot position that could be associated with available inhomogeneity.

Despite general similarity there is a noticeable difference between two spectra shown in Fig. 1: upon qCW excitation the $S$ band becomes asymmetric. We found that this difference is strongly dependent on $I_{\mathrm{exc}}$. To look into this further, we have considered the shape of PL observed at $\mathrm{qCW}$ excitation as a mixture of two functions defined as

$$
\Gamma^{S, L}\left(\lambda, \lambda_{0}^{S, L}, \sigma^{S, L}, \delta^{S, L}\right) \equiv \frac{2}{\sigma^{S, L} \sqrt{2 \pi}\left(2-\delta^{S, L}\right)}\left\{\begin{array}{l}
\exp \left(-\frac{\left(\lambda-\lambda_{0}^{S, L}\right)^{2}}{2\left(\sigma^{S, L}\right)^{2}}\right) \text { if } \lambda>\lambda_{0}^{S, L}, \\
\exp \left(\frac{\left(\lambda-\lambda_{0}^{S, L}\right)^{2}}{2\left(1-\delta^{S, L}\right)^{2}\left(\sigma^{S, L}\right)^{2}}\right) \text { otherwise }
\end{array},\right.
$$

where a convention was established that physical values with $S$ as a superscript will represent the $S$ band, analogously, $L$ will stand for the $L$ band; D $\sigma^{S, L}$ and $0 \leq \delta^{S, L} \leq 1$ are standard deviation and asymmetry parameter, respectively; $\lambda$ and $\lambda_{0}^{S, L}$ represent the current wavelength and emission center of matching luminescence band. Curve fit- ting to a number of spectra measured at different intensities reveals that $\delta^{L}=0$ for any excitation applied (which means that $L$ band is always symmetric) while the asymmetry of the $S$ band disappears at relatively weak laser pulses and saturates for the stronger ones (inset $a$ in Fig. 1). 
Besides above observation, the ratio between heights of two luminescence bands changes slightly with the excitation raise, showing the relative increase of the $L$ band and simultaneous slump of the «blue» shoulder of the $S$ band.

Differences observed point to the same cause that is to the spectral diffusion, though some factors specific to the surface recombination may be also at work. Here some extra words are probably needed to set the link between spectral diffusion and peculiarities observed. Diagnostics of our samples with the technique of scanning electron microscopy (SEM) evidences that close-packed films of nanocrystals rather than separated nanoobjects are formed on the surface of GaAs substrates. (Image of one of these films obtained with SEM technique and 3D-imaging software is shown in inset $b$ in Fig. 1.) These films consist of close-packed nanocrystals with mean diameters falling roughly into two intervals differing by an order of magnitude [4]. In this case the more excitation the more probability to find excited $\mathrm{NC}$ of smaller size in conjunction with bigger ones and therefore the more probability for energy transfer to larger object. Another words with excitation increase e-h pairs will migrate over time from the smaller to larger NC, thereby making the $S$ band asymmetric and pumping the $L$ band. Such migration may even happen when the $s$-shell of the smaller NC is not filled completely [6]. In all fairness one should additionally mention that we observed the weak variations in the intensity ratio of luminescence bands when moving excitation spot over the surface of the given sample, but in this case these changes were random and did not affect the shape of the $S$ band.

Saturation of $\delta^{S}$ value at high laser flux evidences no advance in spectral diffusion at such excitation. However further increase in laser power triggers a dramatic change in PL spectra, i.e., the development of new luminescence band (with center of emission $\lambda_{0}^{M}$ ) denoted as $M$ in Fig. 2. This is clear spectral signature of the multiexcitonic nature of luminescence that arises due to the state filling effect. To analyze the data we used least squares fitting procedure to decompose the measured spectra into a combination of two symmetric (Gaussian, for $L$ and $M$ bands) and one asymmetric ( $\Gamma^{S}$, for the $S$ band) functions, setting $\delta^{S}$ parameter equal to its saturated value (Fig. 2). As the $M$ band is much better resolved at high excitation intensity, the fitting procedure was started at the highest-intensity end of the data set and, then proceeded with decreasing of excitation to the level when $M$ peak was too weak and barely perceptible. It is obvious that results of particular fit may depend on initial values of parameters used. To stabilize the results, the best-fit values of parameters obtained at the higher excitation were used as initial values for the next one. In this way the dependences of integer intensities $\left(I^{S, L, M}\right)$ for $S, L$ and $M$ bands on the level of optical excitation were revealed. Such dependences typical for our samples are shown in

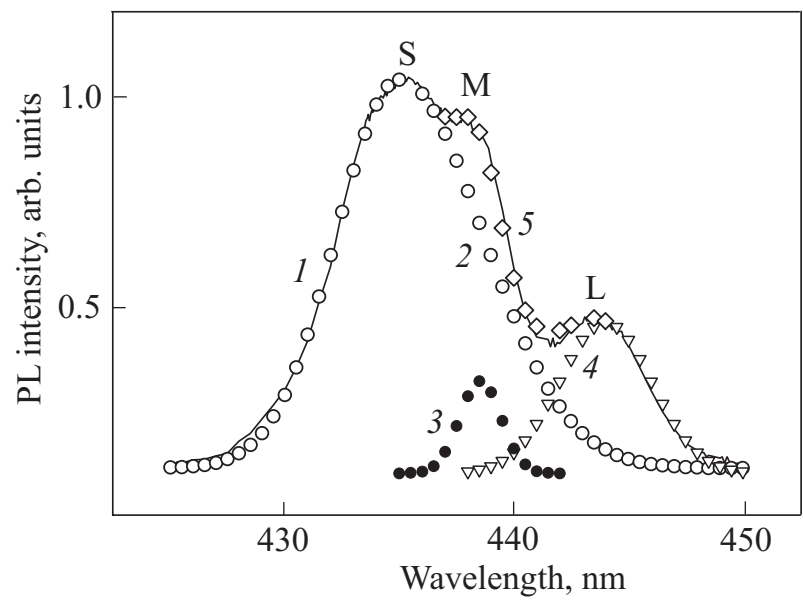

Fig. 2. PL spectrum (1) of ZnSe nanocrystals under exactly focused qCW excitation of relatively high $\left(\sim 10^{23}\right.$ quanta/ $\left.\left(\mathrm{cm}^{2} \cdot \mathrm{s}\right)\right)$ level at $77 \mathrm{~K}$. The spectrum has been normalized to the intensity of $S$ band. Open (2) and filled circles (3) as well as open triangles (4) are results of decomposition of experimental PL spectra (solid line 1) into Gaussians $(3,4)$ and $\Gamma^{S}(2)$ functions with following parameters: $\sigma^{S}=3.65, \sigma^{L}=2.0, \sigma^{M}=0.9, \lambda_{0}^{S}=$ $=435 \mathrm{~nm}, \lambda_{0}^{L}=444 \mathrm{~nm}, \lambda_{0}^{M}=438,5 \mathrm{~nm}$ and $\delta^{S}=0.25$. Open squares (5) represent the fit of $M$ part of experimental spectrum. (Other parts are also perfectly fitted but inevitable picture cluttering prevents this from showing. Limited numbers of 2, 3 and 4 dots are also shown for this reason.)

in Fig. 3. It is obvious that power law of the kind of $I^{S, L, M} \sim I_{\mathrm{exc}}^{\gamma^{S, L, M}}$ provides a fairly good fit to the experimental points - at least within a range of $I_{\text {exc }}$ amounting to two to three orders of magnitude. The general features of the dependences obtained are as follows:

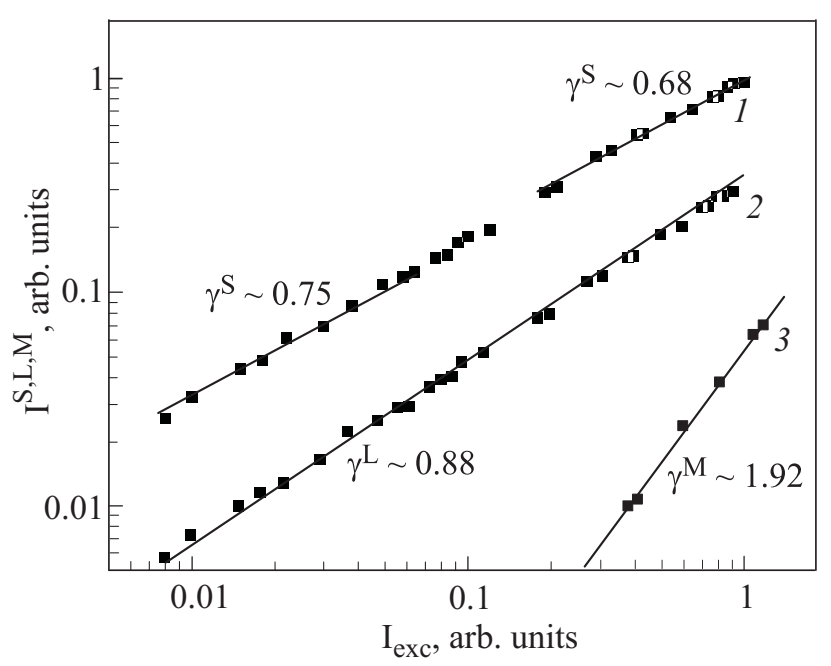

Fig. 3. Emission intensity dependencies of $S(1), L(2)$ and $M$ (3) bands on the level of qCW excitation of ZnSe nanocrystals at $T=77 \mathrm{~K}$. The $\gamma$ values are normalized to the excitation intensity of $10^{23}$ quanta $/\left(\mathrm{cm}^{2} \cdot \mathrm{s}\right)$. Experimental dots are represented by black squares, plots are arbitrary shifted along the $y$ axis and straight lines serve as eyes guidances. The value of $\gamma^{S}, \gamma^{L}$ and $\gamma^{M}$ are indicated. 
First, both $S$ and $L$ bands are sublinear (i.e., $\gamma^{S, L}<1$ ) in the whole range of $I_{\text {exc }}$ employed.

Second, as $I_{\text {exc }}$ becomes larger the deviation from linearity of the $S$ band (i.e., the increase of $\gamma^{S}$ ) grows very gradually suggesting the quenching of band-to-band transitions. This quenching may be due to both surface and Auger recombination and will be studied in details somewhere else by using the technique of femtosecond optical spectroscopy.

Third, the intensity of the emission relevant to the $M$ band increases with approximately quadratic power-law with fitted exponent of $\gamma^{M} \approx 1.92$.

The nearly quadratic power dependence for the $M$ band strongly suggests that it occurrence is due to emission from the QD containing two e-h pairs, that is biexcitons. Such dependence for PL originated from biexcitons in QD was observed for different materials [7-9] and has the transparent physical meaning: the population of biexciton energy level is controlled by two e-h pairs created as a result of two independent processes. The observed deviation from pure quadratic dependence may be due to $\mathrm{e}-\mathrm{h}$ transfer between conjunct $\mathrm{NC}$ in close-packed film and/or nonradiative multiparticle Auger process inside individual QD [10].

$M$ band observed is considered to be related with the $S$ set of NC and broadened inhomogeneously due to its size distributions. From this point one can roughly evaluate the binding energy $(\Delta)$ of biexcitons confined in such nanocrystals. Needless to say, rather broad shapes of PL bands prevent an accurate determination of $\Delta$, so, our estimation is based on difference between $\lambda_{0}^{S}$ and $\lambda_{0}^{M}$. Based on value of this difference and neglecting a possible size-dependence of binding energy for exciton molecule within the range of 3.5-4.0 $\mathrm{nm}$ we argue that $\Delta$ in $\mathrm{ZnSe}$ $\mathrm{NC}$ of relevant sizes may be as large as $23 \mathrm{meV}$. Allowing $\Delta$ to have such a relatively large value we depart from compelling physical argument that forced confinement of free carriers within the same extremely small crystal leads to an enhanced Coulomb attraction between electrons and holes [11,12].

As a final point we would like to comment that any excitation intensities applied was giving no indication of extra band red shifted to $L$ band. This may mean that in the regime of relatively weak confinement plausible for $L$ set the exciton molecules in $\mathrm{ZnSe}$ at $77 \mathrm{~K}$ are not stable enough and PL spectra of relatively large NC are determined by confinement of pure excitons.

In conclusion, we studied PL of $\mathrm{ZnSe}$ nanocrystals with bimodal size distribution in the regime of high excitation by using the qCW laser technique. One of the observed PL bands indicated an efficient formation of biexcitons in NC with characteristic size less than $a_{B}$. This is attributed to an increased Coulomb attraction in totally confined nanostructures. At the same time in $\mathrm{ZnSe}$ NC with a lateral extension far above the $a_{B}$ value the biexcitons seem to be not stable so that no relevant PL was observed.

As an extra result, we have found the significant increase of spectral diffusion in NC under high level of excitation as compared to the regime of low excitation. This is credited to both the close-packed arrangement of NC in samples under the study and the random nature of the excitation process for extremely small objects.

Support of this work by Fundamental Research State Fund of Ukraine ( $\Phi 25.4 / 207$ Project) is gratefully acknowledged.

1. V.I. Klimov, J. Phys. Chem. B110, 16827 (2006).

2. K. Song and S. Lee, Curr. Appl. Phys. 1, 169 (2001).

3. V.V. Nikesh and Sh. Mahamuni, Semicond. Sci. Technol. 16, 1 (2001).

4. V.V. Tishchenko and A. V. Kovalenko, Fiz. Niz. Temp. 32, 1545 (2006) [Low Temp. Phys. 32, 1177 (2006)].

5. H. Hayashi and Sh. Katayama, Phys. Rev. B39, 8743 (1989).

6. M. Grundmann and D. Bimberg, Phys. Rev. B55, 9740 (1997).

7. K. Brunner, G. Abstreiter, G. Böhm, G. Tänkle, and G. Weimann, Phys. Rev. Lett. 73, 1138 (1994).

8. S. Kako, K. Hoshino, S. Iwamoto, S. Ishida, and Y. Arakawa, Appl. Phys. Lett. 85, 64 (2004).

9. R.M. Stevenson, R.M. Thompson, A.J. Shields, I. Farrer, C.J. Lobo, D.A. Ritchie, M.L. Leadbeater, and M. Pepper, Physica E13, 423 (2002).

10. Semiconductor and Metal Nanocrystals: Synthesis and Electronic and Optical Properties, V.I. Klimov (ed.), Marcel Dekker, New York (2003).

11. L. Banyai, Phys. Rev. B39, 8022 (1989).

12. Y.Z. Hu, S.W. Koch, M. Lindberg, N. Peyghambarian, E.L. Pollock, and F.F. Abraham, Phys. Rev. Lett. 64, 1805 (1990). 